\title{
Adherence to antihypertensive medications and health outcomes among newly treated hypertensive patients
}

This article was published in the following Dove Press journal:

ClinicoEconomics and Outcomes Research

5 March 2011

Number of times this article has been viewed

\author{
Luca Degli Esposti' \\ Stefania Saragoni ${ }^{1}$ \\ Silvia Benemei ${ }^{3}$ \\ Paolo Batacchi ${ }^{2}$ \\ Pierangelo Geppetti ${ }^{3}$ \\ Mauro Di Bari ${ }^{3}$ \\ Niccolò Marchionni ${ }^{3}$ \\ Alessandra Sturani ${ }^{4}$ \\ Stefano Buda' \\ Ezio Degli Esposti ${ }^{4}$ \\ 'CliCon S.r.l. Health, Economics and \\ Outcomes Research, Ravenna, Italy; \\ ${ }^{2}$ Pharmaceutical Policy Department, \\ Local Health Unit of Florence, \\ Florence, Italy; ${ }^{3} \mathrm{CIRFF}$, University of \\ Florence, Florence, Italy; ${ }^{4}$ Outcome \\ Research Unit. Policlinics Umberto I, \\ Rome, Italy
}

Correspondence: Luca Degli Esposti CliCon S.r.I.Via Salara, 36. 48I00,

Ravenna, Italy

Tel +39 0544/38393

$\mathrm{Fax}+390544 / 212699$

Email luca.degliesposti@clicon.it
Objective: To evaluate adherence to antihypertensive therapy (AHT) and the association between adherence to AHT, all-cause mortality, and cardiovascular (CV) morbidity in a large cohort of patients newly treated with antihypertensives in a clinical practice setting.

Methods: An administrative database kept by the Local Health Unit of Florence (Italy) listing patient baseline characteristics, drug prescription, and hospital admission information was used to perform a population-based retrospective study including patients newly treated with antihypertensives, $\geq 18$ years of age, with a first prescription between January 1, 2004 and December 31, 2006. Patients using antihypertensives for secondary prevention of CV disease, occasional spot users, and patients with early CV events, were excluded from the study cohort Adherence to AHT was calculated and classified as poor, moderate, good, and excellent. A Cox regression model was conducted to determine the association among adherence to AHT and risk of all-cause mortality, stroke, or acute myocardial infarction.

Results: A total of 31,306 patients, 15,031 men (48.0\%), and 16,275 women (52.0\%), with a mean age of $60.2 \pm 14.5$ years was included in the study. Adherence to AHT was poor in 8038 patients (25.7\% of included patients), moderate in 4640 (14.8\%), good in 5651 (18.1\%), and excellent in 12,977 (41.5\%). Compared with patients with poor adherence (hazard ratio $[\mathrm{HR}]=1$ ), the risk of all-cause death, stroke, or acute myocardial infarction was significantly lower in patients with good $(\mathrm{HR}=0.69, P<0.001)$ and excellent adherence $(\mathrm{HR}=0.53$, $P<0.001)$.

Conclusions: These findings indicate that suboptimal adherence to AHT occurs in a substantial proportion of patients and is associated with poor health outcomes already in primary prevention of CV diseases. For health authorities, this preliminary evidence underlines the need for monitoring and improving medication adherence in clinical practice.

Keywords: antihypertensive drug therapy, adherence, all-cause mortality, stroke, acute myocardial infarction

\section{Introduction}

Clinical trials have shown that continuous treatment with antihypertensive drug therapy (AHT) significantly lowers blood pressure in a very high proportion of patients, thus significantly decreasing both cardiovascular $(\mathrm{CV})$ morbidity and mortality ${ }^{1-4}$ and, ultimately, overall costs associated with high blood pressure..$^{5-7}$

However, in clinical practice, adherence (whether a patient takes medications as prescribed) and persistence (whether a patient remains on therapy as long as needed) to AHT are suboptimal, ${ }^{8-11}$ ranging from $30 \%$ to $70 \%$, depending on measurement methods and definitions. ${ }^{12,13}$ Poor adherence to AHT causes suboptimal blood pressure control and, ultimately, preventable CV morbidity, hospitalizations, mortality, and 
health care expenditure, ${ }^{10,14-16}$ all consequences that have been documented, but still remain overlooked.

The objective of this study was to evaluate adherence to AHT and the association between adherence to AHT, all-cause mortality, and hospitalizations for stroke and acute myocardial infarction (AMI) in a large cohort of patients newly treated with antihypertensives in a clinical practice setting.

\section{Patients and methods}

\section{Data source}

The study was based on administrative databases maintained by the Local Health Unit (LHU) of Florence, Italy, with a population of approximately 800,000 inhabitants. The LHU Ethics Committee approved the study. In the Medications Prescription Database, the LHU routinely measures the volume of expenditure generated by the dispensing of drugs to the enrollees. The data available in each prescription claim include the patient's national health number, the prescribing physician's number, the ATC (anatomical-therapeuticchemical) code of the drug delivered, the number of packs, the number of units per pack, the dosages, the unit cost per pack, and the prescription date. Using the numeric code released to each citizen by the LHU as a unique identifier, this database was linked with the Beneficiaries 'Database, listing some patients' demographic characteristics such date of birth, sex, place of residence, physician licence number, and registration and end of registration date, and the Hospital Discharge Database, which includes all hospitalization data with the discharge diagnosis codes classified according to the International Classification of Diseases, Ninth Revision (ICD 9), and the Mortality Database, where death data are recorded. It was not possible to retrieve the cause of death from death certificates. Universal health-care coverage in Italy allows completeness and comprehensiveness of the information contained in these databases, which have been used in previous epidemiological studies. ${ }^{17}$ The Italian Ministry of Health reported that Tuscany archives are 100\% complete and $95 \%$ accurate. ${ }^{18}$

In order to guarantee patient privacy, each subject was assigned an anonymous univocal alphanumeric code.

\section{Cohort definition}

This was a retrospective cohort study, which included only new AHT users, $\geq 18$ years of age, with a complete history of prescriptions and clinical outcome data over the study period. Subjects were enrolled if they had at least 1 prescription of AHT (diuretics [ATC code C03], excluding loop diuretics [ATC code $\mathrm{C} 03 \mathrm{C}$ ] which are mainly used for heart failure, $\beta$-blockers [ATC code C07], calcium-channel blockers [ATC code C08], angiotensin-converting enzyme inhibitors [ATC code C09A/B], angiotensin-receptor blockers [ATC code C09C/D], or other AHT drugs [ATC code C02]) dispensed between January 1, 2004 and December 31, 2006 (enrolment period). The date of the first AHT claim was defined as the enrolment date. Subjects were defined new users if they had not been prescribed any AHT between January 1, 2002 and the enrolment date.

In order to prevent inclusion of subjects prescribed with any of the index drugs for indications other than hypertension, we excluded subjects who had been diagnosed with heart failure (ICD9 code 428.x), ischemic heart disease (ICD9 code 410 through 414.x), cerebrovascular disorders (ICD9 code 430 through $438 . x$ ), or other CV diseases (ICD9 code 390 through $400 . x, 406$ through 459 excluding the aforementioned diagnosis codes) before the enrolment date and those prescribed with nitrates in the year before the enrolment date. ${ }^{19}$ Furthermore, we also excluded records of subjects who died, moved to other LHU or were hospitalized with a diagnosis of stroke or AMI in the 6 months after the enrolment date, in order to ensure a minimum time interval for adherence assessment: in fact, the use of short-term time intervals to assess adherence has been shown not to reflect accurately long-term behaviors. ${ }^{20,21}$

\section{Adherence to AHT}

Adherence to AHT was estimated as the percentage of days a subject had tablets available (proportion of days covered, PDC), from the first delivery of AHT until either death, moving to another LHU, hospitalization for AMI or stroke, or July 1 , 2007, whichever occurred earlier. The interval was separated into treatment episodes of continuous AHT use based on the method of Catalan and LeLorier. ${ }^{22} \mathrm{~A}$ treatment episode was measured as the time-span between the starting date of the first AHT dispensing until the last day of the final AHT supply. The latter date included allowance for a possible gap after the final dispensing within the specific episode. Prescriptions filled near the end of the interval contributed days till that date.

Prescriptions containing more than 1 drug contributed a) the sum of the days' supply of all drugs, in case of drugs from the same AHT drug class, because a possible stockpiling of medication was considered; b) the lower days' supply drug value, in case of drugs from different AHT drug classes, identifying them as a combined therapy.

The PDC corresponded to the total of number of days' supply of medication dispensed within each episode, 
divided by the total length of the interval and multiplied by 100 . Subjects were grouped into 4 adherence categories as follows: poor ( $\mathrm{PDC} \leq 40 \%)$, moderate (PDC $41 \%$ to $60 \%$ ), good (PDC $61 \%$ to $80 \%$ ), and excellent adherence (PDC $>80 \%$ ). When PDC was $\leq 20 \%$, indication for AHT treatment was considered uncertain or questionable and, therefore, the corresponding subjects were excluded, ${ }^{23}$ of whom $77.2 \%$ received only 1 and $14.6 \%$ only 2 prescriptions.

\section{Outcomes}

Study outcomes were all-cause mortality and the first hospitalization for fatal or nonfatal CV events, represented by a principal discharge diagnosis of AMI (ICD 9 code 410.x) or stroke (ICD 9 codes 430 through 438.x). Patients were followed-up from the date of enrolment until study closure (December 31, 2007) or the date of cancellation from the enrollees' list in the LHU of Florence. Median follow-up duration was 1.9 years, with a maximum of 3.5 (first 6 months after the enrolment date excluded).

\section{Statistical analysis}

Data were summarized as mean \pm standard deviation (SD) for continuous variable and as numbers (percentages) of subjects for categorical variables. Pearson's chi-square and one-way ANOVA tests were used to evaluate differences in baseline characteristics across adherence levels.

Bivariate and multivariable Cox proportional hazard regression models (Table 2, Models 1 and 2, respectively) were used to estimate hazard ratios (HR) and $95 \%$ confidence intervals (CI) of death and CV events, as combined and separate outcomes, as a function of adherence categories. To adjust for potential confounders, in the multivariable model we included age, gender, and presence of specific treatments in the year prior to the enrolment date, taken as proxies for the diagnoses of diabetes mellitus (hypoglycemic agents, ATC code A10), dyslipidemia (hypolipidemic agents, ATC code C10), heart disease (cardiac therapy, ATC code C01, excluding nitrates, ATC code C01DA), and atherosclerotic disease (platelet aggregation inhibitors, ATC $\operatorname{cod} \mathrm{B} 01 \mathrm{AC})$. Visual inspection of the survival curves confirmed that the assumption of proportional hazards was not violated.

Sensitivity analyses were used to assess the robustness of our analysis in the presence of potential biases.

Two-tailed $P$-values $<0.05$ were considered statistically significant. All analyses were conducted using SPSS for Windows, version 15.0.

\section{Results}

Out of an initial selection of 63,027 records of a new prescription for AHT agents, 31,721 (50.3\%) were excluded because of hospitalization for $\mathrm{CV}$ disease prior to enrolment $(2,896,4.6 \%)$, use of nitrates in the previous year (539, $0.9 \%$ ), cancellation from the LHU list or occurrence of a study outcome in the 6 months after enrolment $(1269,2.0 \%)$, or PDC $\leq 20 \%(27,017,42.8 \%)$. Thus, 31,306 patients $(15,031$ men, $48.0 \%)$ were included, whose mean age was $60.2 \pm 14.5$ years (range 18 to 101 years). Use of hypoglycemic agents, lipid-modifying agents, cardiac therapy, and platelet aggregation inhibitors is shown in Table 1.

\section{Adherence to AHT}

Adherence to AHT was poor in 8038 patients subjects $(25.7 \%)$, moderate in $4640(14.8 \%)$, good in $5651(18.1 \%)$, and excellent in 12,977 (41.5\%) (Table 1). Poorly adherent patients were younger, predominantly women, and with lower prevalence of associated hypoglycemic, lipid-lowering, and platelet aggregation inhibitor agents (Table 1), whereas use of cardiac therapy was limited, uncommon, and unrelated to adherence level.

Table I Characteristics of subjects newly treated with antihypertensive medications, by level of adherence to treatment

\begin{tabular}{|c|c|c|c|c|c|c|}
\hline & \multicolumn{5}{|c|}{ Adherence $^{\mathrm{a}}$} & \multirow[t]{2}{*}{ Total } \\
\hline & Poor & Moderate & Good & Excellent & $P$ value & \\
\hline Subjects, n (\%) & $8038(25.7)$ & $4640(\mid 4.8)$ & $565 I(\mid 8.1)$ & $12977(4 \mid .5)$ & & $31306(100.0)$ \\
\hline Age, mean (SD), years & $57.3(17.3)$ & $60.3(15.2)$ & $61.6(13.5)$ & $6 \mathrm{I} .4(12.5)$ & $<0.001$ & $60.2(14.5)$ \\
\hline Male (\%) & 43.6 & 43.9 & 45.0 & 53.5 & $<0.001$ & 48.0 \\
\hline \multicolumn{7}{|l|}{ Associated drug therapy ${ }^{\mathrm{b}}$} \\
\hline Hypoglycemic agents (\%) & 4.4 & 5.2 & 5.6 & 7.0 & $<0.00 \mathrm{I}$ & 5.8 \\
\hline Lipid lowering agents (\%) & 4.3 & 4.6 & 4.9 & 5.3 & 0.007 & 4.9 \\
\hline Cardiac therapy (\%) & 1.3 & 1.7 & 1.3 & 1.6 & 0.190 & 1.5 \\
\hline Platelet aggregation inhibitors (\%) & 5.8 & 6.1 & 7.2 & 7.1 & $<0.001$ & 6.6 \\
\hline
\end{tabular}

Notes: a Defined on the basis of the proportion of days covered (PDC): poor, PDC $\leq 40 \%$; moderate, PDC $41 \%$ to $60 \%$; good, PDC $61 \%$ to $80 \%$; excellent, PDC > $80 \%$. 'One year before the enrolment date. 
Table 2 Adherence to antihypertensive medications and risk of the combined outcome of all-cause death, stroke, or acute myocardial infarction, estimated by Cox proportional hazards models

\begin{tabular}{|c|c|c|c|c|}
\hline & \multicolumn{2}{|l|}{ Model I } & \multicolumn{2}{|l|}{ Model 2} \\
\hline & HR (95\% Cl) & $P$ value & HR (95\% Cl) & $P$ value \\
\hline Adherence $^{a}$ & & $<0.001^{\mathrm{a}}$ & & $<0.00 \mathrm{I}^{\mathrm{b}}$ \\
\hline Poor & 1.00 & & 1.00 & \\
\hline Moderate & $0.94(0.80-1.1 \mathrm{I})$ & 0.464 & $0.87(0.74-1.03)$ & 0.104 \\
\hline Good & $0.77(0.65-0.90)$ & 0.001 & $0.69(0.58-0.8 \mathrm{I})$ & $<0.001$ \\
\hline Excellent & $0.61(0.53-0.70)$ & $<0.001$ & $0.53(0.46-0.6 \mathrm{I})$ & $<0.001$ \\
\hline \multicolumn{5}{|l|}{ Age, years } \\
\hline$<45$ & - & - & 1.00 & \\
\hline $45-65$ & - & - & $2.67(1.84-3.88)$ & $<0.001$ \\
\hline$>65$ & - & - & $10.48(7.3|-| 5.03)$ & $<0.001$ \\
\hline \multicolumn{5}{|l|}{ Gender } \\
\hline Male & - & - & 1.00 & \\
\hline Female & - & - & $0.62(0.55-0.69)$ & $<0.001$ \\
\hline \multicolumn{5}{|l|}{ Associated drug therapy ${ }^{c, d}$} \\
\hline Hypoglycemic agents & - & - & $1.67(1.4 \mathrm{I}-1.99)$ & $<0.001$ \\
\hline Lipid-lowering agents & - & - & $0.70(0.54-0.91)$ & 0.006 \\
\hline Cardiac therapy & - & - & $1.69(1.29-2.23)$ & $<0.001$ \\
\hline Platelet aggregation inhibitors & - & - & $1.66(1.42-1.93)$ & $<0.001$ \\
\hline
\end{tabular}

Notes: Bivariate and multivariable risks are shown in Models I and 2, respectively; a total of 1263 events in 31,306 subjects was considered in the models; ${ }^{2}$ defined as in Table I; bfor trend; 'absence of medication as reference; 'one year before the enrolment date.

Abbreviations: $\mathrm{Cl}$, confidence interval; $\mathrm{HR}$, hazard ratio.

\section{Health outcomes}

Out of 31,306 patients, a total of 1263 (4.0\%) combined endpoints was seen in the follow-up, including 768 deaths, 370 strokes, and 191 AMI. Incidence of the combined endpoint decreased significantly with increasing adherence, being 29.5, 24.8, 19.6, and 16.1 per 1000 person-years in patients whose adherence was poor, moderate, good, and excellent, respectively. The corresponding bivariate HRs are shown in Table 2, Model 1. This association remained unaffected when adjusting for covariates in multivariable Model 2, Table 2 and Figure 1: the risk of the combined endpoint increased stepwise with decreasing adherence as well as with advancing age, was greater in males and in users of hypoglycemic agents or platelet aggregation inhibitors or cardiac therapy, and lower in the presence of treatment with lipid-lowering agents.

The results obtained for the individual endpoints in separate analyses were similar for the outcome of all-cause mortality, as shown by $P$ for trend values (Table 3 and Figure 2). The analyses for the outcomes of stroke and AMI showed no statistically significant differences between individual levels of adherence and the study outcome (Table 3 and Figures 3 and 4).

\section{Sensitivity analysis}

To examine the robustness of our results, we did several additional analyses.
First, to ensure that our method did not introduce a survival bias, we re-estimated the association between adherence-study outcomes among subjects surviving and free of AMI or stroke for at least 3 months and 1 year after the enrolment date.

Second, the primary categorization of adherence based on PDC level: poor (PDC $\leq 40 \%$ ), moderate (PDC, $41 \%$ to $60 \%$ ), good (PDC, $61 \%$ to $80 \%$ ), excellent (PDC $>80 \%$ ) was

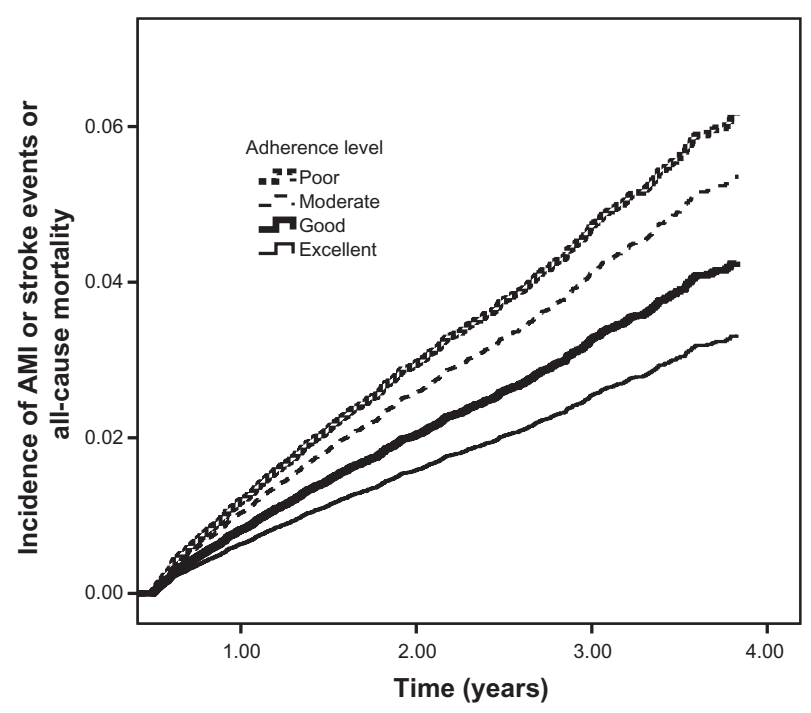

Figure I Combined endpoint of all-cause death, stroke, or acute myocardial infarction (AMI) curves in 3I,340 subjects newly treated with antihypertensive therapy, by levels of adherence to treatment. 
Table 3 Adherence to antihypertensive medications and risk of all-cause death, stroke, and acute myocardial infarction, estimated in separate multivariable Cox proportional hazards models

\begin{tabular}{|c|c|c|c|c|c|c|}
\hline & \multicolumn{2}{|l|}{ All-cause death } & \multicolumn{2}{|l|}{ Stroke } & \multicolumn{2}{|c|}{ Acute myocardial infarction } \\
\hline & HR (95\% Cl) & $P$ value & HR (95\% CI) & $P$ value & HR (95\% CI) & $P$ value \\
\hline Adherence $^{a}$ & & $<0.00 \mathrm{I}^{\mathrm{b}}$ & & $0.38 I^{b}$ & & $0.877^{\mathrm{b}}$ \\
\hline Poor & 1.00 & & 1.00 & & 1.00 & \\
\hline Moderate & $0.8 \mathrm{I}(0.66-0.99)$ & 0.038 & $0.96(0.69-1.33)$ & 0.799 & I.I4 (0.7I-I.83) & 0.592 \\
\hline Good & $0.59(0.48-0.72)$ & $<0.001$ & $0.81(0.59-1.11)$ & 0.197 & $0.98(0.62-1.55)$ & 0.939 \\
\hline Excellent & $0.37(0.3 \mathrm{I}-0.45)$ & $<0.001$ & $0.82(0.63-1.07)$ & 0.138 & $0.96(0.65-1.40)$ & 0.825 \\
\hline
\end{tabular}

Notes: Totals of 768 deaths in 30,668 subjects and 370 strokes and I9I acute myocardial infarctions in 31,340 subjects were considered in the models; $\mathrm{HRs}$ with $95 \%$ Cls adjusted for age, gender, and use of hypoglycemic agents, lipid-lowering agents, cardiac therapy, and platelet aggregation inhibitors before the enrolment date; adefined as in Table I; bfor trend.

Abbreviations: $\mathrm{Cl}$, confidence interval; $\mathrm{HR}$, hazard ratio.

arbitrary. However, we examined the effect of medication adherence on outcomes using different definitions of PDC cutoffs: quartiles, $\leq 25 \%, 26 \%$ to $50 \%, 51 \%$ to $75 \%,>75 \%$.

Third, as previous studies described the relationship between CV disease severity factors, level of adherence to AHTs and, consequently, the development of adverse outcome events, ${ }^{19,23,24}$ to minimize the bias, the adherencestudy outcomes association was also evaluated across different risk subgroups: older patients ( $>65$ years), patients with at least 1 medication evaluated in the year before the enrolment date.

The results of these additional analyses were consistent with our primary findings. Data are shown in Tables 4 and 5.

\section{Discussion}

Despite the considerable magnitude and the remarkable consequences of suboptimal adherence to AHT shown in randomized controlled trials as well as in observational studies, ${ }^{1-3}$ in clinical practice, evidence of the association

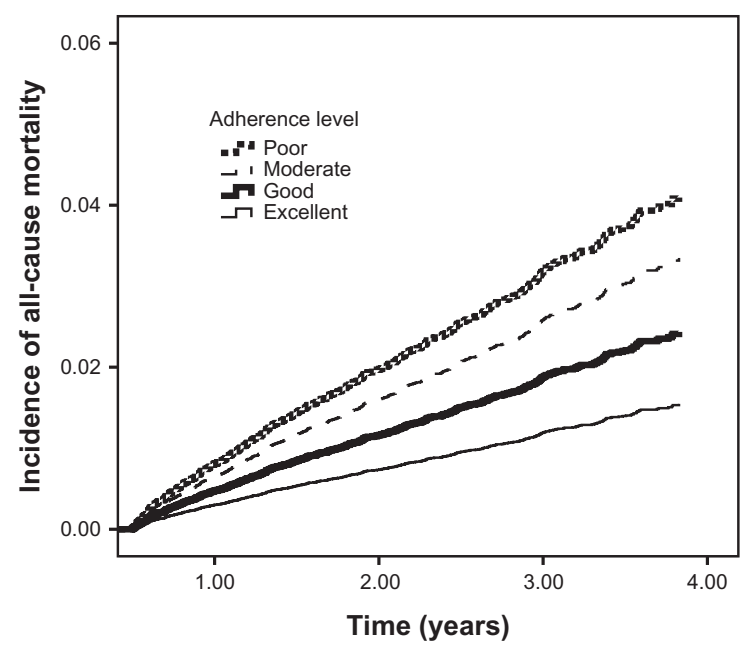

Figure 2 Incidence of all-cause mortality among new antihypertensive therapy users. between adherence to AHT, all-cause mortality, and CV morbidity is still limited. Existing publications focus on newly diagnosed hypertensive patients, diabetics, and patients with previous $\mathrm{CV}$ events - that is, mostly on secondary prevention. Studied outcomes include CV hospitalizations (mainly in primary prevention studies ${ }^{19,23,24}$ ), and all-cause hospitalizations or all-cause mortality (mainly in secondary prevention studies ${ }^{20,25-27}$ ). The present study analyzed allcause mortality, and the first occurrence of stroke and AMI among hypertensive patients newly treated with AHT, in primary prevention.

The risk of all-cause mortality, stroke, and AMI decreased progressively as adherence to AHT increased. Compared with poor adherence, the risk of experiencing a major health outcome decreased stepwise from good ( $31 \%$ lower risk) to excellent (47\% lower risk) (Table 2). The risk gradient did not attain statistical significance in subjects with moderate adherence, thus possibly suggesting some treatment effectiveness threshold. This is an original finding of the present study, due to the large number of records available,

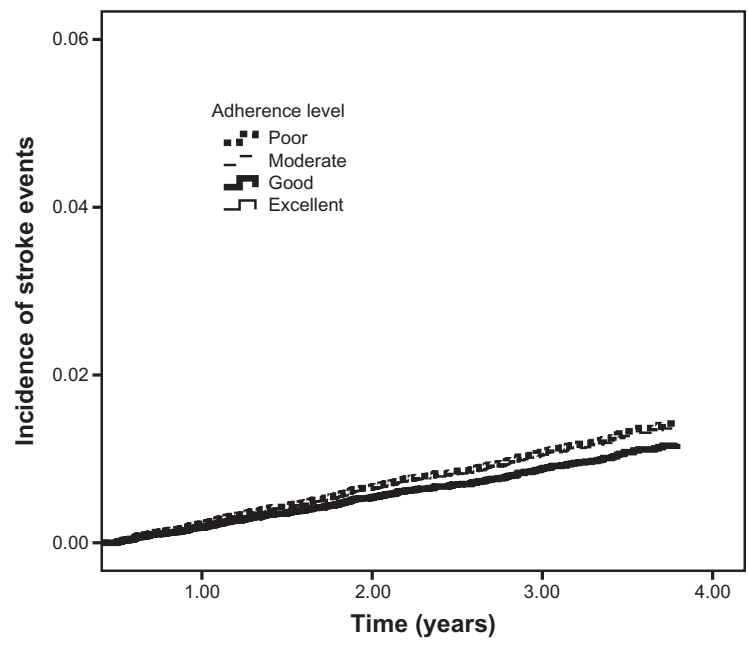

Figure 3 Incidence of stroke events among new antihypertensive therapy users. 


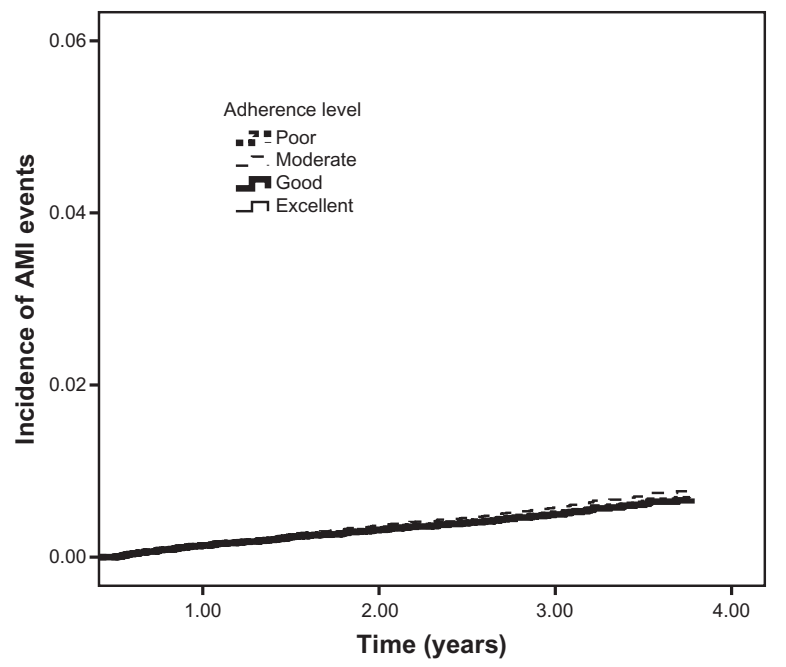

Figure 4 Incidence of acute myocardial infarction (AMI) events among new antihypertensive therapy users.

which enabled the definition of 4 levels of adherence, whereas most of the previous studies referred to just 2 levels of adherence. ${ }^{19,23}$ As this is an observational study, the allocation of patients into different adherence groups was not randomized and it might have produced study groups not similar in their baseline $\mathrm{CV}$ risk profile. So this bias might have altered the observed reduction of the occurrence of events associated with increased adherence. However, the results obtained after controlling for baseline differences in the regression model and stratifyng the patient population into different risk subgroups in the sensitivity analisys confirmed the robustness of our findings.

In separate analyses of the individual endpoints, the risk associated with poor adherence was significant for allcause mortality and directionally similar for both stroke and AMI, though with lower, nonsignificant gradients. Thus, the present study shows that adherence to AHT is associated with a substantial reduction in mortality risk also in primary prevention, whereas previous findings were restricted to secondary prevention..$^{20,25,26}$ In the analysis of the occurrence of each single health outcome, the reduction in the risk of stroke and AMI was lower than that reported by Breekveldt-Postma et al. ${ }^{19}$ However, in our study, the duration of the follow-up was much shorter (median of 1.9 years in our study versus up to a maximum of 10 years) and the observed number of strokes (370 versus 1293) and AMI (191 versus 826) was lower. Another study, recently published by Mazzaglia et al, reported greater risk reduction with increasing adherence. Compared with the present study, the endpoint of the study by Mazzaglia et al was a combination of a wider range of CV events, not only stroke and AMI, and the follow-up available was longer and extended up to 4.6 years. ${ }^{24}$ Previous studies with almost the same follow-up duration (2.4 years) as ours reported higher risk reduction only in highest-risk patients (eg, patients with a previous episode of $\mathrm{AMI}^{20}$ ). Nevertheless, the favorable trend in reducing $\mathrm{CV}$ events is more evident for nonfatal stroke than nonfatal AMI, thus confirming that for causespecific events AHT is associated with a major reduction in the risk of fatal or nonfatal stroke, but coronary events are reduced as well, though to a lesser degree. ${ }^{6}$

\section{Limitations}

Several potential limitations of this study should be considered.

First, there was a lack of clinical data. The present study was conducted by cross-linking Medications Prescriptions Database with Health-assisted Subjects' Database, Hospital Discharge Database, and Mortality Database. These ad hoc databases, comparable to the healthcare claim databases that have been utilized for years in United States and Canada for outcome research, ${ }^{28,29}$ were originally constructed to serve a billing role, the reimbursement for services provided.

Table 4 One-way sensitivity analysis for the risk of the combined outcome of all-cause death, stroke, or acute myocardial infarction

\begin{tabular}{|c|c|c|c|c|c|c|}
\hline & \multicolumn{6}{|l|}{ Adherence $^{a}$} \\
\hline & \multicolumn{2}{|l|}{ Moderate } & \multicolumn{2}{|l|}{ Good } & \multicolumn{2}{|l|}{ Excellent } \\
\hline & HR $(95 \% \mathrm{CI})$ & $P$ value & HR (95\% Cl) & $P$ value & HR $(95 \% \mathrm{Cl})$ & $P$ value \\
\hline Absence of study outcomes for at least 3 months & $0.94(0.81-1.08)$ & 0.374 & $0.70(0.60-0.8 \mathrm{I})$ & $<0.001$ & $0.55(0.50-0.64)$ & $<0.001$ \\
\hline Absence of study outcomes for at least I year & $0.87(0.71-1.07)$ & 0.196 & $0.72(0.59-0.88)$ & 0.001 & $0.56(0.47-0.67)$ & $<0.001$ \\
\hline Adherence categories: quartiles ${ }^{b}$ & $0.88(0.72-1.07)$ & 0.152 & $0.73(0.64-0.83)$ & $<0.001$ & $0.5 I(0.43-0.59)$ & $<0.001$ \\
\hline Adherence categories: $\leq 25,26-50,5 \mathrm{I}-75,>75 \%{ }^{\mathrm{c}}$ & $0.83(0.68-1.01)$ & 0.058 & $0.72(0.59-0.88)$ & 0.002 & $0.50(0.40-0.59)$ & $<0.001$ \\
\hline Age $>65$ & $0.85(0.71-1.03)$ & 0.100 & $0.67(0.55-0.79)$ & $<0.001$ & $0.52(0.44-0.61)$ & $<0.001$ \\
\hline At least I comorbidity & $0.96(0.84-1.13)$ & 0.417 & $0.70(0.55-1.02)$ & 0.059 & $0.54(0.49-0.70)$ & 0.006 \\
\hline
\end{tabular}

Notes: aCases of proportion of days covered (PDC) $\leq 20 \%$ were excluded; bthe first quartile corresponds to the poor category of adherence, the second quartile to the moderate category, the third quartile to the good category, the fourth quartile to the excellent category; CPDC $\leq 25 \%$ corresponds to the poor category, PDC $26 \%$ to $50 \%$ to moderate, PDC $51 \%$ to $75 \%$ to good, PDC $>75 \%$ to excellent.

Abbreviations: $\mathrm{Cl}$, confidence interval; $\mathrm{HR}$, hazard ratio. 
Table 5 One-way sensitivity analysis for the risk of all-cause death

\begin{tabular}{|c|c|c|c|c|c|c|}
\hline & \multicolumn{6}{|l|}{ Adherence $^{\mathrm{a}}$} \\
\hline & \multicolumn{2}{|l|}{ Moderate } & \multicolumn{2}{|l|}{ Good } & \multicolumn{2}{|l|}{ Excellent } \\
\hline & HR (95\% Cl) & $P$ value & HR (95\% CI) & $P$ value & HR (95\% CI) & $P$ value \\
\hline Absence of study outcomes for at least 3 months & $0.86(0.72-1.03)$ & 0.095 & $0.60(0.50-0.72)$ & $<0.001$ & $0.39(0.35-0.48)$ & $<0.001$ \\
\hline Absence of study outcomes for at least I year & $0.81(0.63-1.04)$ & 0.098 & $0.62(0.48-0.79)$ & $<0.001$ & $0.39(0.33-0.5 \mathrm{I})$ & $<0.001$ \\
\hline Adherence categories: quartiles ${ }^{b}$ & $0.79(0.53-1.07)$ & 0.103 & $0.61(0.54-0.75)$ & $<0.00 \mathrm{I}$ & $0.36(0.30-0.42)$ & $<0.001$ \\
\hline Adherence categories: $\leq 25,26-50,5 \mathrm{I}-75,>75 \% \mathrm{c}$ & $0.84(0.66-1.06)$ & 0.140 & $0.60(0.5 I-0.78)$ & 0.001 & $0.35(0.28-0.45)$ & $<0.00$ I \\
\hline Age $>65$ years & $0.83(0.64-1.07)$ & 0.148 & $0.57(0.4 I-0.73)$ & $<0.001$ & $0.36(0.31-0.45)$ & $<0.001$ \\
\hline At least I comorbidity & $0.75(0.50-1.12)$ & 0.162 & $0.59(0.47-0.7 \mathrm{I})$ & 0.001 & $0.40(0.29-0.68)$ & $<0.001$ \\
\hline
\end{tabular}

Notes: acases of proportion of days covered (PDC) $\leq 20 \%$ were excluded; b the first quartile corresponds to the poor category of adherence, the second quartile to the moderate category, the third quartile to the good category, the fourth quartile to the excellent category; cPDC $\leq 25 \%$ corresponds to the poor category, PDC $26 \%$ to $50 \%$ to moderate; PDC $51 \%$ to $75 \%$ to good, PDC $>75 \%$ to excellent.

Abbreviations: $\mathrm{Cl}$, confidence interval; $\mathrm{HR}$, hazard ratio.

Thus, information on clinical data was not available and, as a consequence, the adjustment for blood pressure levels was not permitted.

However, the confounding effect of baseline blood pressure on the association between adherence to AHT agents and $\mathrm{CV}$ events is probably limited, as suggested by a recent study in a general practice population, where baseline systolic and diastolic blood pressure values were comparable between low-, intermediate-, and high-adherence patients. ${ }^{24}$ Moreover, a recent meta-analysis of 147 trials $^{30}$ showed a benefit in lowering blood pressure by antihypertensive drugs whatever the patients' blood pressure was, thus avoiding the need to measure blood pressure routinely.

Second, a formal diagnosis of arterial hypertension was not available. In the present study, indeed, the diagnosis was surrogated by prescription of AHT, thus leaving room for potential misclassification, as only some of the subjects treated with AHT drugs may have been truly hypertensive. However, the exclusion of subjects with only 1 or 2 prescriptions and of those with previous hospitalizations or therapies for $\mathrm{CV}$ diseases should have minimized this misclassification.

Third, the measurement of drug use was based on dispensed medications and actual drug-taking behaviors remain unknown. Empirical evidence suggests, however, a strong correlation between pharmacy claims and drug exposure. ${ }^{31,32}$

Fourth, information bias may also have been possible. As hospital discharge information was obtained from the Florence Hospital Discharge Database, we could not track hospitalizations collected in other Italian LHUs' databases.

Finally, the data source utilized did not allow for lifestyle adjustments, such as physical activity, smoking, and other determinants of $\mathrm{CV}$ morbidity and mortality. The potential confounding effect of these variables on the association between adherence and $\mathrm{CV}$ events should be analyzed in future studies.

\section{Conclusions}

The findings from the present study indicate that suboptimal adherence to AHT is associated with an avoidable number of all-cause deaths and hospitalizations for CV disease in primary prevention. In a public health perspective, this evidence underlines the need for monitoring and improving medications adherence in clinical practice. Studies based on administrative databases, like the present one, offer several advantages, including a prompt, easily updated, and representative picture of the monitored cohorts for a prolonged duration of observation, with highly generalizable results.

Consequences of poor adherence with AHT extend beyond health prevention and involve costs for $\mathrm{CV}$ prevention and economic sustainability of national health services. Although the therapeutic benefits of AHT are well understood, the potential economic advantages are often overlooked in the public debate, which is dominated by concerns about escalating expenditure for prescription drugs. Increased drug utilization can provide a net economic return when it is driven by improved adherence to guidelines-based therapy. ${ }^{16}$ Although drug costs are a relatively small percentage of total healthcare costs, ${ }^{33,34}$ they have high leverage - a small increase in drug costs (associated with improved adherence) can produce a much larger reduction in the general economic burden for health.

\section{Acknowledgments}

This study was funded by Takeda Italia Farmaceutici. The cooperation of Dr Alessandra Fionda is gratefully acknowledged.

\section{Disclosure}

None of the authors declare any conflict of interest and all persons who contributed to the work meet the criteria for authorship. 


\section{References}

1. Psaty BM, Lumley T, Furberg CD, et al. Health outcomes associated with various antihypertensive therapies used as first-line agents: a network meta analysis. JAMA. 2003;289:2534-2544.

2. Blood Pressure Lowering Treatment Trialists' Collaboration. Effects of different blood pressure lowering regimens on major cardiovascular events: second cycle of prospectively designed overviews. Lancet. 2003; 362:1527-1535.

3. Chalmers J. Comparison of various blood pressure lowering treatments on the primary prevention of cardiovascular outcomes in recent randomised clinical trials. Clin Exp Hypertens. 2004;26:709-719.

4. Turnbull F, Neal B, Ninomiya T, et al. Effects of different regimens to lower blood pressure on major cardiovascular events in older and younger adults: meta-analysis of randomised trials. Blood Pressure Lowering Treatment Trialists' Collaboration. BMJ. 2008;336:1121-1123.

5. American Heart Association. High Blood Pressure 2009. http://www. americanheart.org/presenter.jhtml. Accessed April 24, 2009.

6. Hansson L, Lloyd A, Anderson P, et al. Excess morbidity and cost of failure to achieve targets for blood pressure control in Europe. Blood Press. 2002;11:35-45.

7. Gaziano TA, Bitton A, Anand S, et al. The global cost of non optimal blood pressure. J Hypertens. 2009;27:1472-1477.

8. Degli Esposti L, Valpiani G. Pharmacoeconomic burden of undertreating hypertension. Pharmacoeconomics. 2004;22:907-928.

9. Costa FV, D'Ausilio A, Bianchi C, et al. Adherence to anti-hypertensive medications: a review and update. High Blood Pressure and Cardiovascular Prevention. 2009;16:101-110.

10. Fitz-Simon N, Bennett K, Feely A. A review of studies of adherence with antihypertensive drugs using prescription databases. Ther Clin Risk Manag. 2005;1:93-106.

11. Burnier M. Medication adherence and persistence as the cornerstone of effective antihypertensive therapy. Am J Hypertens. 2006;19: 1190-1196.

12. Andrade SE, Kahler KH, Frech F, et al. Methods for evaluation of medication adherence and persistence using automated databases. Pharmacoepidemiol Drug Safety. 2006;15:565-574.

13. ISPOR Medication Compliance Special Interest Group. http://www. ispor.org/sigs/medication.asp. Accessed 23 June, 2005.

14. Elliott WJ. Improving outcomes in hypertensive patients: focus on adherence and persistence with antihypertensive therapy. J Clin Hypertens. 2009; 11:376-382.

15. Muszbek N, Brixner D, Benedict A, et al. The economic consequences of non-compliance in cardiovascular disease and related conditions: a literature review. Int J Clin Pract. 2008;62:338-351.

16. Sokol MC, McGuigan KA, Verbrugge RR, et al. Impact of medication adherence on hospitalization risk and healthcare cost. Med Care. 2005; 43:521-530.

17. Di Bari M, Balzi D, Roberts AT, et al. Prognostic stratification o folder persons based on simple administrative data: development and validation of the "Silver Code", to be used in emergency department triage. J Gerontol A Biol Sci Med. 2010;65:159-164.
18. Ministero del Lavoro, della Salute e delle Politiche Sociali. Rapporto annuale sulla attività di ricovero ospedaliero. Anno 2005. http:// www.ministerosalute.it/programmazione/sdo/sezDocumenti. jsp?id=148\&label=osp. Accessed April 24, 2009.

19. Breekveldt-Postma NS, Penning-van Beest FJ, Siiskonen SJ, et al. The effect of discontinuation of antihypertensives on the risk of acute myocardial infarction and stroke. Curr Med Res Opin. 2008;24: 121-127.

20. Rasmussen JN, Chong A, Alter DA. Relationship between adherence to evidence-based pharmacotherapy and long-term mortality after acute myocardial infarction. JAMA. 2007;297:177-186.

21. Suissa S. Immortal time bias in observational studies of drug effects. Pharmacoepidemiol Drug Safety. 2007;16:241-249.

22. Catalan VS, LeLorier J. Predictors of long-term persistence on statins in a subsidized clinical population. Value Health. 2000;3:417-426.

23. Kettani FZ, Dragomir A, Cŏté R, et al. Impact of a better adherence to antihypertensive agents on cerebrovascular disease for primary prevention. Stroke. 2009;40:213-220.

24. Mazzaglia G, Ambrosioni E, Alacqua M, et al. Adherence to antihypertensive medications and cardiovascular morbidity among newly diagnosed hypertensive patients. Circulation. 2009;120:1598-1605.

25. Ho PM, Rumsfeld JS, Masoudi FA, et al. Effect of medication nonadherence on hospitalization and mortality among patients with diabetes mellitus. Arch Intern Med. 2006;166:1836-1841.

26. Ho PM, Spertus JA, Masoudi FA, et al. Impact of medication therapy discontinuation on mortality after myocardial infarction. Arch Intern Med. 2006;166:1842-1847.

27. Gislason GH, Rasmussen JN, Abildstrom SZ, et al. Persistent use of evidence-based pharmacotherapy in heart failure is associated with improved outcomes. Circulation. 2007;116:737-744.

28. Motheral BR, Fairman KA. The use of claims databases for antihypertensive drugs and associated hospitalization. Clin Ther. 1997;19: 346-366.

29. Birnbaum HG, Cremieux PY, Greenberg PE, et al. Using health care expenditures for healthcare claims data for outcome research and pharmaco-economic analyses. Pharmacoeconomics. 1999;16:1-8.

30. Law MR, Morris JK, Wald NJ. Use of blood pressure lowering drugs in the prevention of cardiovascular disease: meta-analysis of 147 randomised trials in the context of expectations from prospective epidemiological studies. BMJ. 2009;338:b1665.

31. Elliott WJ, Plauschinat CA, Skrepnek GH, Gause D. Persistence, adherence, and risk of discontinuation associated with commonly prescribed antihypertensive drug monotherapies. J Am Board Fam Med. 2007;20:72-80.

32. Elliott WJ. Compliance strategies. Curr Opin Nephrol Hypertens. 1994; 3:271-278.

33. Leal J, Luengo-Fernandez R, Gray A. Economic burden of cardiovascular diseases in the enlarged European Union. Eur Heart J. 2006;27: 1610-1619.

34. Tarride JE, Lim M, DesMeules M, et al. A review of the cost of cardiovascular disease. Can J Cardiol. 2009;25:e195-e202.
ClinicoEconomics and Outcomes Research

\section{Publish your work in this journal}

ClinicoEconomics \& Outcomes Research is an international, peerreviewed open-access journal focusing on Health Technology Assessment, Pharmacoeconomics and Outcomes Research in the areas of diagnosis, medical devices, and clinical, surgical and pharmacological intervention. The economic impact of health policy and health systems

\section{Dovepress}

organization also constitute important areas of coverage. The manuscript management system is completely online and includes a very quick and fair peer-review system, which is all easy to use. Visit $\mathrm{http}: / /$ www.dovepress.com/testimonials.php to read real quotes from published authors. 